\title{
Automatic Detection of CT Perfusion Datasets Unsuitable for Analysis due to Head Movement of Acute Ischemic Stroke Patients
}

\author{
Fahmi Fahmi ${ }^{1,3 *}$, Henk A. Marquering1, ${ }^{1,}$, Geert J. Streekstra ${ }^{1,2}$, Ludo F.M. \\ Beenen $^{2}$, Natasja N.Y. Janssen ${ }^{1}$, Charles B.L. Majoie ${ }^{2}$ and Ed vanBavel ${ }^{1}$ \\ ${ }^{1}$ Department of Biomedical Engineering and Physics, \\ ${ }^{2}$ Department of Radiology, Academic Medical Centre, Amsterdam, the Netherlands \\ ${ }^{3}$ Department of Electrical Engineering, University of Sumatra Utara, Indonesia
}

Submitted June 2013. Accepted for publication October 2013.

\begin{abstract}
Head movement during brain Computed Tomography Perfusion (CTP) can deteriorate perfusion analysis quality in acute ischemic stroke patients. We developed a method for automatic detection of CTP datasets with excessive head movement, based on 3D image-registration of CTP, with non-contrast CT providing transformation parameters. For parameter values exceeding predefined thresholds, the dataset was classified as 'severely moved'. Threshold values were determined by digital CTP phantom experiments. The automated selection was compared to manual screening by 2 experienced radiologists for 114 brain CTP datasets. Based on receiver operator characteristics, optimal thresholds were found of respectively $1.0^{\circ}, 2.8^{\circ}$ and $6.9^{\circ}$ for pitch, roll and yaw, and $2.8 \mathrm{~mm}$ for z-axis translation. The proposed method had a sensitivity of $91.4 \%$ and a specificity of $82.3 \%$. This method allows accurate automated detection of brain CTP datasets that are unsuitable for perfusion analysis.
\end{abstract}

Keywords: brain CT perfusion, computer aided detection, acute ischemic stroke, patient movement

\section{INTRODUCTION}

Brain Computed Tomography Perfusion (CTP) imaging is evolving towards a promising diagnostic tool for initial evaluation of acute ischemic stroke patients [1-4]. In CTP images, areas with brain perfusion defects can be detected after the onset of clinical symptoms and can facilitate the differentiation between the irreversibly damaged infarct core and the potentially salvageable penumbra [5-9]. Such differentiation is important in choosing the most suitable therapy [10-12].

The patient's head movement during image acquisition, however, limits the applicability of CTP. Since CTP analysis assumes that a specific location in the images is associated with a single anatomical position [13,14], it is likely that head movement deteriorates CTP analysis results, as shown by an example in Figure 1.

\footnotetext{
*Corresponding Author: Fahmi Fahmi, MSc, Academic Medical Center (AMC), Department of Biomedical Engineering and Physics, L0-147, Meibergdreef 9, 1105 AZ Amsterdam, The Netherlands, Phone: +31(020)5665189, Fax: +31(020)6917233, E-mail: f.fahmi@amc.uva.nl.

Other authors:h.a.marquering@amc.uva.nl, g.j.streekstra@amc.uva.nl, 1.f.beenen@amc.uva.nl, n.n.janssen@amc.uva.nl, c.b.majoie@amc.uva.nl,e.vanbavel@amc.uva.nl.
} 
(a)

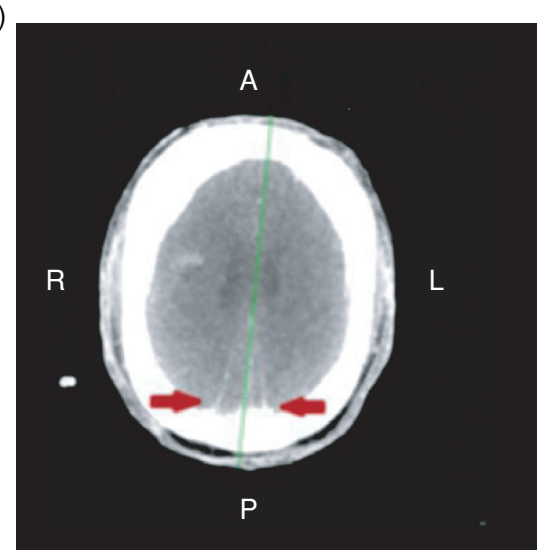

(b)

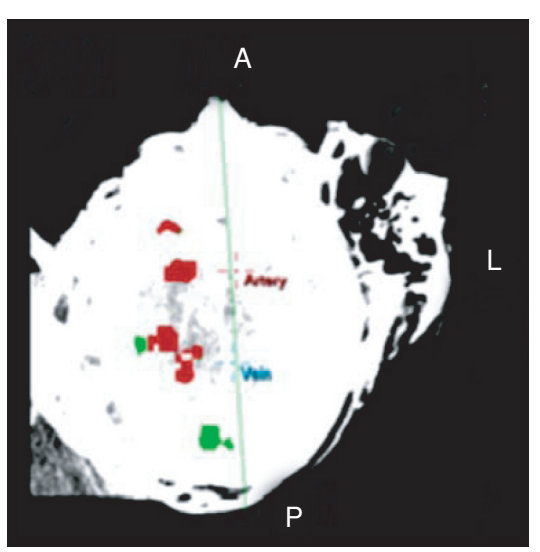

Figure 1. Example of CTP analysis disturbed by head movement:(a) The red arrows indicate double falx cerebri due to moderate head movement. (b) An example of deterioration of brain CTP images due to severe head movement.

While it is indeed well recognized that head movement is a common problem during CTP image acquisition of stroke patients [15-18], automated procedures for exclusion of unsuited CTP data are lacking, and substantial manual work by radiologists is required.

In current clinical practice, a radiologist must first visually inspect the CTP datasets in order to decide whether they are affected by head movement, and whether the data set is suitable for accurate perfusion analysis. If the data set passes such inspection, basic registration, commonly available in software analysis packages, is applied to correct for small head motion. Alternatively, certain time frames of the CTP acquisition that could disturb the perfusion analysis can be removed. The visual inspection is performed for all 25-30 time frames and is commonly conducted in 2D maximum intensity projections. In addition to the time requirement, selection of out-of plane head movement acquisitions with this method is sometimes difficult. Furthermore, there is no common consensus on selection criteria for CTP data that would be suitable for analysis. Therefore, a considerable inter-observer variation can be expected.

The goal of this study is to develop and validate an automatic detection method for unsuitable CTP images due to excessive head movement.

\section{METHODS}

This study utilized results from previous phantom experiments to estimate threshold values for motion parameters beyond which unacceptable deviations of perfusion analysis results occurred [19]. Head movement was quantitatively assessed using a 3D image registration with non-contrast $\mathrm{CT}$ (ncCT). Based on the comparison of motion parameters with threshold values, we labeled CTP data as "no or minimal" or "severe" movement. Finally, we validated this automated detection of CTP data of severe movement with manual screening by radiologists to define the optimum threshold values. 


\subsection{Estimation of Threshold Values Based on Phantom Experiments}

To define threshold values for movement beyond which severe deterioration of CTP quality can be expected, we utilized results from a previous study on the effect of head movement on CTP analysis [19]. In that study, CTP digital hybrid phantom data developed by Riordan et al. [20] were employed.

The phantom was based on a combination of CT images of an anthropomorphic head phantom with clinically acquired high resolution 7T clinical MRI images. It included healthy and diseased brain parenchyma, as well as the cerebral vascular system. Time attenuation curves emulating contrast bolus passage based on perfusion as observed in CTP clinical studies were added [20]. The infarct volume used in that study was positioned at the right part of the brain, where the proportional volume of infarct core and penumbra were created by applying a mask to the digital hybrid phantom data. To simulate head movement, the CTP phantom data were rotated around and translated along each coordinate axis. The original and transformed phantom datasets were then processed using Extended Brilliance Workspace version 3.5 Brain CT Perfusion Package (Philips Healthcare, Cleveland, $\mathrm{OH}$ ), as the standard software package used in our clinical and research practice. Summary maps were subsequently generated. These maps utilize all perfusion maps, and quantitatively describe both infarct core and penumbra area.

The volume similarity and the spatial agreement between summary maps generated from the original and rotated phantom data were calculated. The agreement was expressed with the Dice Similarity Coefficient (DSC), a measurement of spatial overlap of image regions. DSC is defined as twice the volume of the intersection of two areas divided by the sum of the volume of both areas. DSC expresses agreement not only in the size but also in the location of infarct core and penumbra between summary maps [19].

\subsubsection{Threshold Estimation}

Rotation and translations values relating to given DSC values were used as potential threshold. We explored DSC values ranging from 0.4 to 0.8 to obtain candidate threshold parameter sets for automated motion detection. In our experiments, we excluded the in-plane translations of $\mathrm{x}$ - and $\mathrm{y}$-directions because the phantom study showed that the CTP analysis software can correct these translations; therefore, these translations do not have an effect on the result. One set of threshold values therefore consists of roll, pitch and yaw rotation angle, and translation in z-direction.

\subsection{Head Movement Measurement in Clinical CTP Data}

\subsubsection{CTP Image Acquisition}

We collected 114 CTP data sets from 100 patients suspected of acute ischemic stroke, who underwent ncCT and CTP in our medical centre during 2010-2012. All CT image acquisitions were performed on a 64 slice Siemens scanner (Somatom Sensation 64, Siemens Medical Systems, Erlangen Germany). This retrospective analysis of anonymous patient data was approved by the medical ethics committee of our medical centre. Informed consent was waived because no diagnostic tests other than the routine

clinical imaging were involved in this study. Because the evaluation of the images for the purpose of the current study was performed retrospectively, its results could not influence clinical decisions. 
For the CTP acquisition, $40 \mathrm{ml}$ iopromide (Ultravist 320; Bayer HealthCare Pharmaceuticals, Pine Brook, New Jersey) was infused at $4 \mathrm{ml} / \mathrm{s}$ using an 18 gauge canula in the right antecubital vein, followed by $40 \mathrm{ml} \mathrm{NaCl} 0.9 \%$ bolus. Acquisition and reconstruction parameters were $80 \mathrm{kV}$ tube voltage, $150 \mathrm{mAs}$, collimation $24 \infty 1.2$ $\mathrm{mm}$, field of view $300 \mathrm{~mm}$, reconstructed slice width of $4.8 \mathrm{~mm}$ with 25 time frames acquired. During acquisition, a standard foam headrest was used to provide patient with comfortable position and to minimize the head movement. The ncCT scan of the brain was acquired at admission with $120 \mathrm{kV}$ and 300-375 mAs. This scan covers the whole brain, with a reconstructed slice thickness of $5 \mathrm{~mm}$ or smaller.

\subsubsection{Quantification of Head Movement}

Range and direction of head movement were quantified by the 3D registration of every time frame in the CTP dataset with the ncCT image data of the same patient [18]. The rigid registration resulted in 6 motion parameters: three angles of rotations: pitch $(\mathrm{Rx})$, roll (Ry), and yaw (Rz); and three spatial translations in the x-, y- and z-directions (Tx, Ty, and Tz) (Figure 2). The registration was performed using Elastix [21, 22], with the normalized correlation coefficient as a similarity measure and the use of the gradient descent algorithm to solve for the optimization.

The extent of movement was determined for each time frame, providing $25 \infty 6$ temporal motion parameter values, using the first time frame as a reference.

\subsection{Manual Screening}

Two radiologists (co-authors CBLM and LFMB both with more than 10 years of experience) qualitatively graded the severity of the patient's head movement in CTP

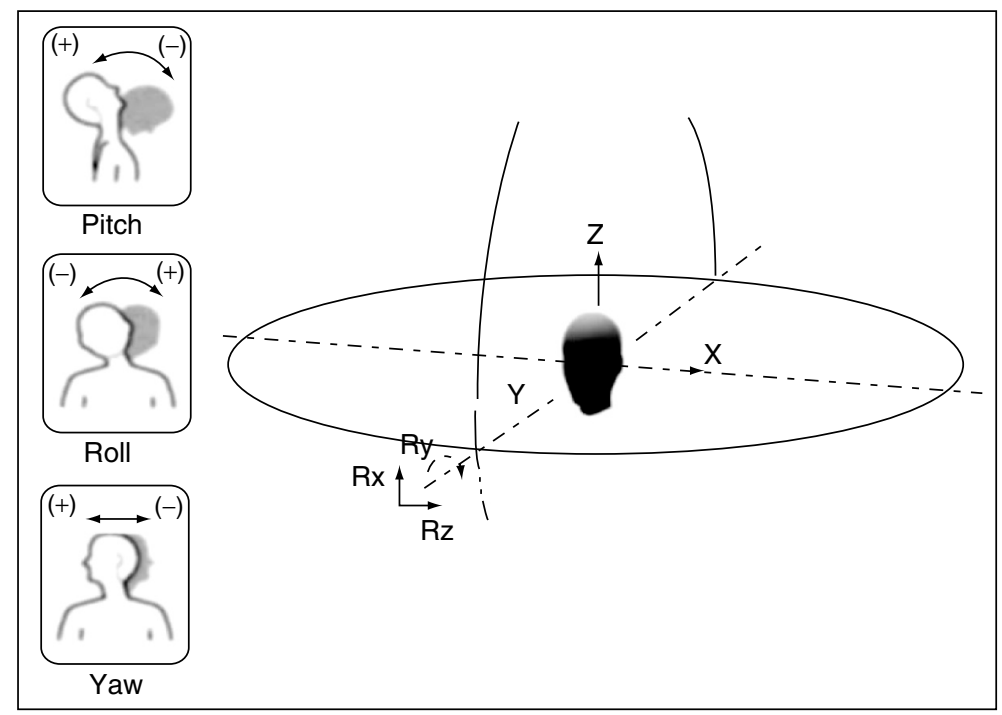

Figure 2. Head movement parameters. Rx is the rotation around the $\mathrm{x}$-axis (pitch), $\mathrm{Ry}$ is the rotation around the $\mathrm{y}$-axis (roll), and $\mathrm{Rz}$ is the rotation around the z-axis (yaw). 
data as 'no or minor' head movement, or as "severe" when the CTP source images showed movement that was expected to severely affect the CTP analysis. A consensus reading was conducted where both radiologists together inspected the source data for any head movement. When necessary, the cine mode was employed for this purpose. The decisions were based on agreement between both radiologists. This manual screening of CTP data was used as the ground truth for the validation of the automatic detection.

\subsection{The Automated Detection}

The decision to label a CTP dataset as "no or minimal" versus "severe" head movement was based on the comparison of quantified motion parameters with the estimated threshold values. If one or more of these motion parameters exceeded the threshold value, it was labeled as "severe"; otherwise the CTP dataset was labeled as "no or minimal" head movement.

\subsection{Statistical Analysis}

We used a binomial classification test in the comparison of the automatic detection and the manual screening to evaluate the performance of the automated method. For each set of threshold values, we determine the true positive rate (TP), for cases when both the automatic method and the manual screening judged the CTP dataset as "severe"; false positive rate (FP) for cases when the automatic method labeled CTP dataset as "severe", but the manual screening did not; true negative rate (TN), when both the automatic method and the manual screening judged the CTP dataset as "no or minimal"; and false negative rate (FN), when the automatic method labeled CTP dataset as "no or minimal", but the manual screening did not. Diagnostic accuracy, sensitivity and specificity of this automatic method were calculated for each set of threshold values with 95\% confident interval. Positive predictive value (PPV) and negative predictive value (NPV) as well as the predictive power (PP) were calculated to describe the performance of the test. Furthermore, receiver-operating characteristics (ROC) for different sets of threshold values were calculated.

\section{RESULTS}

Among the 114 CTP datasets from 100 patients analyzed during manual screening, the radiologists judged movement in 35 CTP dataset as "severe" and the remaining 79 CTP datasets as having "no or minimal" movement.

\subsection{Threshold Values}

The deterioration of CTP analysis with increasing motion in the digital phantom study is illustrated in Figure 3, in which the DSC is plotted as a function of motion parameters. We derived 5 sets of threshold values at DSC values of 0.4, 0.5, 0.6, 0.7, and 0.8. This figure shows that different types of head movement lead to different effects on the accuracy of the perfusion analysis result. For example, it took more than $6^{\circ}$ for yaw rotation $(\mathrm{Rz})$ to cause deterioration, whereas a pitch rotation of $1^{\circ}$ already had a significant deteriorative effect on the CTP analysis results.

The rotation and translation thresholds for each selected DSC value are listed in Table 1. These threshold values were calculated by taking the average of two 
Dice similarity coefficient (DSC)

(a)

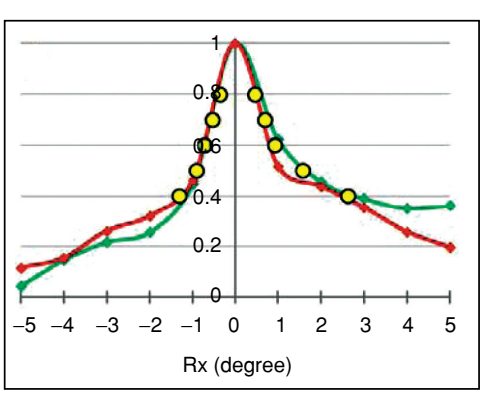

(c)

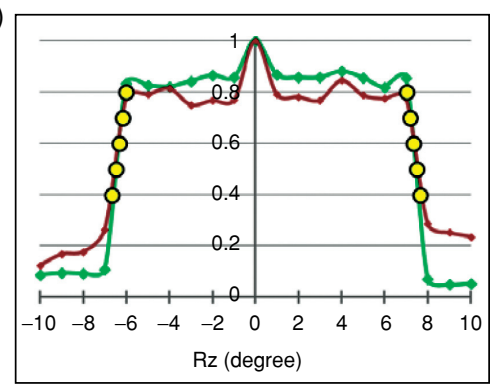

(b)

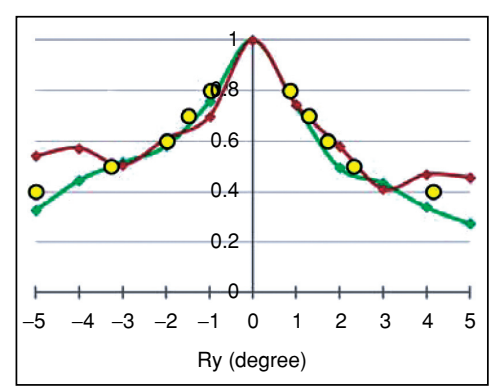

(d)

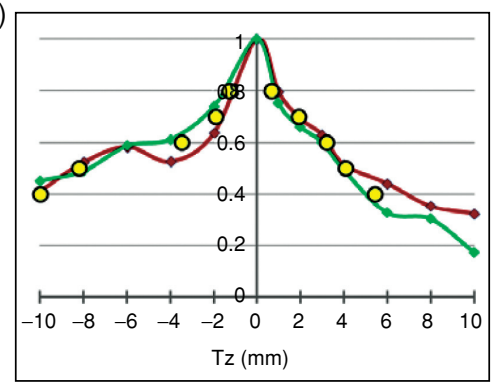

Core

Prenumbra

Interpolation

Figure 3. DSC graph indicating the accuracy of the CTP analysis in a moving phantom. The red line describes the accuracy of the infarct core volume estimation; the green line depicts the accuracy of the infarct penumbra (adapted from [19]). The yellow dots represent interpolation for given DSC values, indicating the threshold values for roll (a), pitch (b), and yaw (c) rotation angles, and the translation in the $\mathrm{z}$ - direction (d). The $\mathrm{y}$ axis for each of the 4 figures is DSC (non-dimensional).

Table 1. Threshold values of the motion parameters associated with specific DSC values

\begin{tabular}{lcccc}
\hline DSC value & Rx $[\mathbf{d e g}]$ & Ry $[\mathbf{d e g}]$ & $\mathbf{R z}[\mathbf{d e g}]$ & $\mathbf{T z}[\mathbf{m m}]$ \\
\hline 0.4 & 1.97 & 5.01 & 7.15 & 8.14 \\
0.5 & 1.04 & 2.80 & 6.99 & 6.14 \\
0.6 & 0.83 & 1.85 & 6.83 & 3.34 \\
0.7 & 0.62 & 1.39 & 6.68 & 1.93 \\
0.8 & 0.42 & 0.92 & 6.52 & 0.98
\end{tabular}

corresponding values for negative and positive displacements in the DSC graph. The translations in the $\mathrm{x}$ - and $\mathrm{y}$ - directions are omitted here because the CTP analysis software is insensitive to in-plane movement. 


\subsection{Quantization of Head Movement}

In our population of 100 patients, the mean rotation angles were $1.7 \pm 3.0^{\circ}, 2.1 \pm 6.2^{\circ}$ and $3.3 \pm 7.7^{\circ}$ for pitch, roll and yaw, respectively. The mean translation was $2.2 \pm 4.1$ $\mathrm{mm}, 1.2 \pm 2.0 \mathrm{~mm}$ and $1.6 \pm 2.2 \mathrm{~mm}$ for $\mathrm{x}-, \mathrm{y}$ - and z-directions, respectively. The largest rotation recorded was in-plane movement (yaw) with maximum rotation angle of $61.3^{\circ}$. Figure 4 shows an example of the dynamics of head movement parameters during the CTP acquisition.

\subsection{Accuracy}

For each DSC value, the automated detection of severe head movement was performed and compared with the manual screening, as shown in Table 2. In this comparison, the manual screening was taken as the ground truth. The diagnostic accuracy was calculated for 5 sets of motion thresholds associated with the DSC values (see Table 1 for the specific motion thresholds).

The ROC curve is shown in Figure 5 with an area under curve of 0.94 . The optimal threshold DSC was 0.5 (predictive power 0.79 ), with an accuracy of $85.1 \%$ (95\%CI: $78.6 \%-91.6 \%)$, sensitivity of $91.4 \%(95 \% \mathrm{CI}: 86.3 \%-96.5 \%)$ and a specificity of $82.3 \%$ (95\% CI: $75.3 \%-89.3 \%)$.
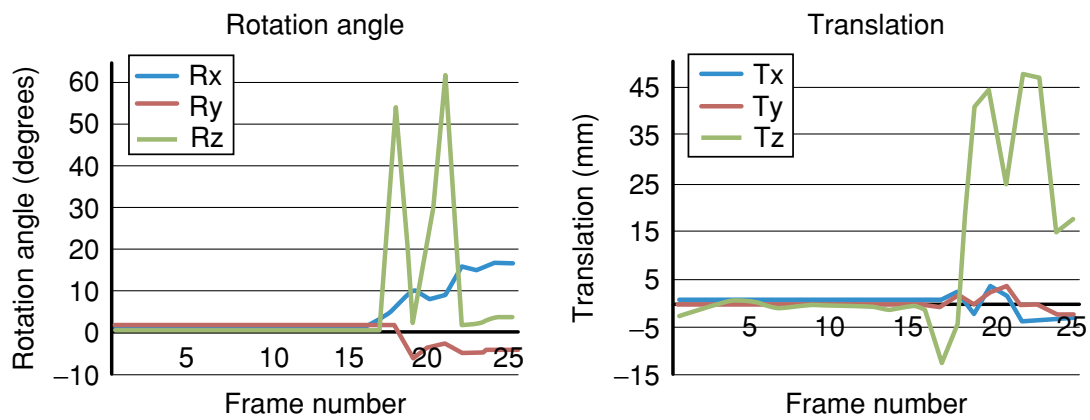

Figure 4. Example of head movement parameters: rotations (left) and translations (right) for 25 time frames as a result of head movement quantization. This figure indicates that there is a large yaw rotation and z-translation around the $17^{\text {th }}$ time frame.

Table 2. Diagnostic accuracy of the automated detection method for different DSC values

\begin{tabular}{lcccccccccc}
\hline DSC value & TP & TN & FP & FN & PPV & NPV & Sensitivity & Specificity & Accuracy & PP \\
\hline 0.4 & 24 & 76 & 3 & 11 & $88.9 \%$ & $87.4 \%$ & $68.6 \%$ & $96.2 \%$ & $87.7 \%$ & 0.77 \\
0.5 & 32 & 65 & 14 & 3 & $69.6 \%$ & $95.6 \%$ & $91.4 \%$ & $82.3 \%$ & $85.1 \%$ & 0.79 \\
0.6 & 35 & 54 & 25 & 0 & $58.3 \%$ & $100 \%$ & $100 \%$ & $68.4 \%$ & $78.1 \%$ & 0.74 \\
0.7 & 35 & 44 & 35 & 0 & $50.0 \%$ & $100 \%$ & $100 \%$ & $55.7 \%$ & $69.3 \%$ & 0.67 \\
0.8 & 35 & 28 & 51 & 0 & $40.7 \%$ & $100 \%$ & $100 \%$ & $35.4 \%$ & $55.3 \%$ & 0.58
\end{tabular}




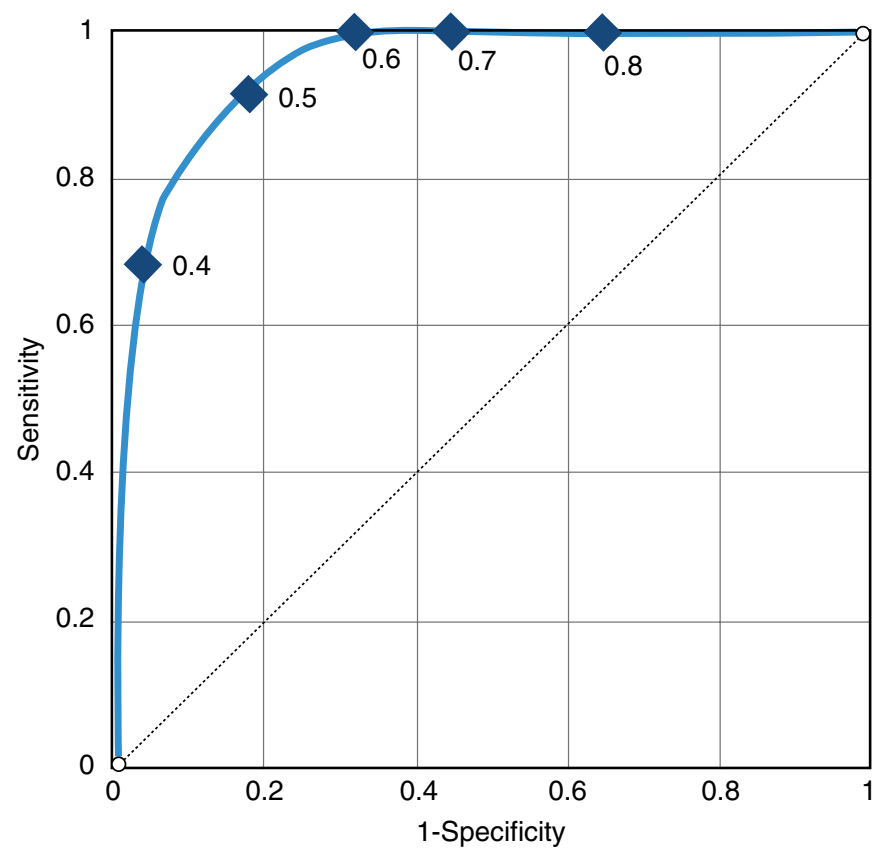

Figure 5. ROC curve (area under curve $=0.94$ ) for different set of threshold values from DSC value of 0.4 to 0.8 .

\section{DISCUSSION}

We presented a method for automatic detection of unsuitable CTP datasets based on the quantification of head movement between time frames. These head movement parameters were compared to threshold values based on simulations of CT perfusion analysis with a CTP phantom data. Performance of this automatic detection method, as evaluated by comparison with manual classification of head movement severity by radiologists, was high (sensitivity and specificity of $91.4 \%$ and $82.3 \%$, respectively).

It was shown previously that standard CTP analysis software can only correct head movement for a limited range of movement. Moreover, such correction is limited to in plane movement only [19]. Head movement strongly alters estimated size and position of infarct core and penumbra produced by CTP analysis. The range of head movement parameters with an acceptable accurate CTP analysis with DSC value 0.5 were $1.0^{\circ}$ for pitch, $2.8^{\circ}$ for roll and $6.9^{\circ}$ for yaw; and $2.8 \mathrm{~mm}$ translation in the z-direction. These values were used as threshold values to identified CTP data as having "no or minimal" or "severe" movement. These threshold values might be different for other software packages. Similar phantom studies should therefore also be conducted for other software packages in order to allow adequate automatic detection of motion-affected CTP analyses. 
The ROC analysis provided an optimal threshold at DSC value of 0.5 . The sensitivity of the proposed method for this threshold is high, while the specificity is somewhat lower. This characteristic is preferable for a computer aided system as presented here, where the radiologists are expected to check the suspected CTP data. For this DSC value, the automatic detection resulted in an overestimation of suspicious image data compared to the selection of the radiologists. For a higher DSC value of 0.6 , the sensitivity of the automated detection could be raised to $100 \%$ at the cost of a specificity decrease to $68 \%$. The threshold values used in this study could be optimized for other CTP methods and scanners. However, this was beyond the scope of this study.

There are several reasons to choose ncCT as a reference target for 3D registration. An ncCT scan was always performed for patients suspected of stroke [2, 23, 24]; therefore, no additional scan was required for the registration. It covers the whole brain and is scanned in less than a second, a much shorter time period than the CTP acquisition which requires up to 50 seconds. Furthermore, there is typically no, or only minimal, image degradation due to head movement during the ncCT acquisition. If severe head movement occurs during ncCT scanning and is noticed by the technician, ncCT can be easily repeated.

The current automatic detection method is limited to the detection of head movement between two time frames and ignores motion that may occur during the acquisition of a particular time frame. Analysis of the effects of such in-frame motion artifacts was beyond the scope of this study.

By quantifying all head movement parameters for each time frame as shown in Figure 3, this method is able to identify culprit time frames that need to be removed for better CTP analysis quality. However, sufficient dynamical information is required and it is therefore not clear to what extent frames can be removed without severely affecting the CTP analysis. Future work is needed to study this subject in more detail.

We need to emphasize that CTP datasets labeled as "severe movement" are not necessarily useless in the sense that all data should automatically be discarded and excluded from analysis. Rather, such a label should warn the radiologists to check the dataset for its usability. For dataset labeled as "no or minimal movement", the radiologist can skip the manual inspection and immediately continue with the CTP analysis, saving substantial time.

During the analysis, we did not consider the moment of the head movement during the whole period of wash-in and wash-out of the contrast agent. Motion artefacts may cause larger deterioration of the analysis when they occur during the passage of the bulk of the contrast as compared to early after injection or during the distal tail of the contrast passage. Nevertheless, additional research on this issue is needed.

This study has some limitations. First, the required computational time needed for the automatic detection is currently too long for introduction in clinical practice. Time constraint is an important issue especially in patients with acute ischemic stroke [25-27]. The most time consuming process of this study was the simulation of CTP phantom data in order to derive the threshold values for the head movement parameters. Once the threshold values were determined, only rigid registrations of the whole CTP 
datasets with ncCT image were required, which still took up to 15-20 minutes. This latter process could be expedited with more computer resources. The aim of this study was to provide a proof of concept rather than a clinical application, therefore reduction of computation time was beyond the scope of this study. Second, the used motion threshold values were derived from a CTP phantom instead of large clinical datasets. An intrinsic difficulty of using clinical datasets for threshold optimization is the absence of a ground truth. The use of CTP phantom data for this purpose seems therefore more suitable. Third, our study was based only on expert qualitative grading and may lead to subjectivity of the evaluation. Therefore, the high percentage of CTP dataset labeled as "severe" could probably be due to the selection bias. Despite of its limitation, manual screening was the best reference we have for validation. Furthermore, we tried to reduce subjectivity by using the evaluation of two radiologists instead of one.

The presented method has the potential to be integrated in clinical practice such that any movement during CTP acquisition will be automatically detected, allowing radiologists to remove suspicious image data from the CTP analysis. The same method can be extended by correction of the head movement, and we are currently studying such possibilities.

\section{CONCLUSION}

We presented a method that automatically selects CTP datasets with severe head movement. The accuracy of the method was $85.1 \%$ with a high sensitivity $(91.4 \%)$ and a good specificity (82.3\%). It supports accuracy assurance of CTP analysis and avoids clinical decisions based upon faulty image data due to severe head movement.

\section{ACKNOWLEDGEMENTS}

This work was supported by LP3M University of Sumatera Utara and RS Pendidikan USU through Directorate General of Higher Education (DIKTI) Scholarship, Ministry of National Education Indonesia.

\section{CONFLICT OF INTEREST}

All authors indicated no potential conflict of interest.

\section{REFERENCES}

[1] M. H. Lev. Perfusion imaging of acute stroke: its role in current and future clinical practice. Radiology, 2013, 266(1):22-7.

[2] G. Zhu, P. Michel, A. Aghaebrahim, J. T. Patrie, W. Xin, A. Eskandari, W. Zhang, and M. Wintermark. Computed Tomography Workup of Patients Suspected of Acute Ischemic Stroke: Perfusion Computed Tomography Adds Value Compared With Clinical Evaluation, Noncontrast Computed Tomography, and Computed Tomography Angiogram in Terms of Predicting Outcome. Stroke, 2013, 44(4):1049-55.

[3] A. R. Hochberg, and G. S. Young. Cerebral perfusion imaging. Semin Neurol, 2013, 32(4):454-65.

[4] B. C. Campbell, L. Weir, P. M. Desmond, H. T. Tu, P. J. Hand, B. Yan, G. A. Donnan, M. W. Parsons, and S. M. Davis. CT perfusion improves diagnostic accuracy and confidence in acute ischaemic stroke. J Neurol Neurosurg Psychiatry, 2013, 84(6):613-8.

[5] C. S. Kidwell, M. Wintermark, D. A. De Silva, T. J. Schaewe, R. Jahan, S. Starkman, T. Jovin, J. Hom, M. Jumaa, J. Schreier, J. Gornbein, D. S. Liebeskind, J. R. Alger, and J. L. Saver. Multiparametric MRI 
and CT Models of Infarct Core and Favorable Penumbral Imaging Patterns in Acute Ischemic Stroke. Stroke, 2013, 44(1):73-9.

[6] F. Zhu, D. Rodriguez Gonzalez, T. Carpenter, M. Atkinson, and J. Wardlaw. Lesion Area Detection Using Source Image Correlation Coefficient for CT Perfusion Imaging. Biomedical and Health Informatics, IEEE Journal of, 2013, PP(99):1-1.

[7] C. Y. Ho, S. Hussain, T. Alam, I. Ahmad, I. C. Wu, and D. P. O’Neill. Accuracy of CT cerebral perfusion in predicting infarct in the emergency department: lesion characterization on CT perfusion based on commercially available software. Emerg Radiol, 2013, 20(3):203-12.

[8] B. N. Huisa, W. P. Neil, R. Schrader, M. Maya, B. Pereira, N. T. Bruce, and P. D. Lyden. Clinical Use of Computed Tomographic Perfusion for the Diagnosis and Prediction of Lesion Growth in Acute Ischemic Stroke. J Stroke Cerebrovasc Dis, 2014, 23(1):114-22.

[9] A. Bivard, C. Levi, N. Spratt, and M. Parsons. Perfusion CT in Acute Stroke: A Comprehensive Analysis of Infarct and Penumbra. Radiology, 2013, 267(2):543-50.

[10] T. Shang, and D. R. Yavagal. Application of acute stroke imaging: selecting patients for revascularization therapy. Neurology, 2012, 79(13):S86-94.

[11] N. Janjua. Use of neuroimaging to guide the treatment of patients beyond the 8-hour time window. Neurology, 2012, 79(13 Suppl 1):S95-9.

[12] A. S. Turk, J. A. Magarick, D. Frei, K. M. Fargen, I. Chaudry, C. A. Holmstedt, J. Nicholas, J. Mocco, R. D. Turner, D. Huddle, D. Loy, R. Bellon, G. Dooley, R. Adams, M. Whaley, C. Fanale, and E. Jauch. CT perfusion-guided patient selection for endovascular recanalization in acute ischemic stroke: a multicenter study. J Neurointerv Surg, 2013, 5(6):523-7.

[13] M. Wintermark, R. Sincic, D. Sridhar, and J. D. Chien. Cerebral perfusion CT: technique and clinical applications. J Neuroradiol, 2008, 35(5):253-60.

[14] A. Cianfoni, C. Colosimo, M. Basile, M. Wintermark, and L. Bonomo. Brain perfusion CT: principles, technique and clinical applications. Radiol Med, 2007, 112(8):1225-43.

[15] M. Sesay, A. Jeannin, C. T. Moonen, V. Dousset, and P. Maurette. Pharmacological control of head motion during cerebral blood flow imaging with CT or MRI. J Neuroradiol, 2009, 36(3):170-3.

[16] X. C. Wang, P. Y. Gao, Y. Lin, L. Ma, r. Guan, J. Xue, B. B. Sui, C. J. Wang, and Y. J. Wang. Clinical value of computed tomography perfusion source images in acute stroke. Neurol Res, 2009, 31(10):1079-83.

[17] S. F. Halpin. Brain imaging using multislice CT: a personal perspective. Br J Radiol, 2004, 77 Spec No 1: S20-6.

[18] F. Fahmi, L. F. Beenen, G. J. Streekstra, N. Y. Janssen, H. W. de Jong, A. Riordan, Y. B. Roos, C. B. Majoie, E. Vanbavel, and H. A. Marquering. Head movement during CT brain perfusion acquisition of patients with suspected acute ischemic stroke. Eur J Radiol, 2013, 82(12): 2334-41.

[19] F. Fahmi, A. Riordan, L. F. Beenen, G. J. Streekstra, N. Y. Janssen, H. W. de Jong, C. B. Majoie, E. van Bavel, and H. A. Marquering. The effect of head movement on CT perfusion summary maps: simulations with CT hybrid phantom data. Med Biol Eng Comput, 2013, 52(2):141-7.

[20] A. J. Riordan, M. Prokop, M. A. Viergever, J. W. Dankbaar, E. J. Smit, and H. W. de Jong. Validation of CT brain perfusion methods using a realistic dynamic head phantom. Med Phys, 2011, 38(6):3212-21.

[21] S. Klein, M. Staring, K. Murphy, M. A. Viergever, and J. P. Pluim. elastix: a toolbox for intensity-based medical image registration. IEEE Trans Med Imaging, 2010, 29(1):196-205.

[22] K. Murphy, B. van Ginneken, J. M. Reinhardt, S. Kabus, K. Ding, X. Deng, K. Cao, K. Du, G. E. Christensen, V. Garcia, T. Vercauteren, N. Ayache, O. Commowick, G. Malandain, B. Glocker, N. Paragios, N. Navab, V. Gorbunova, J. Sporring, M. de Bruijne, X. Han, M. P. Heinrich, J. A. Schnabel, M. Jenkinson, C. Lorenz, M. Modat, J. R. McClelland, S. Ourselin, S. E. Muenzing, M. A. Viergever, D. De Nigris, D. L. Collins, T. Arbel, M. Peroni, R. Li, G. C. Sharp, A. Schmidt-Richberg, J. Ehrhardt, R. Werner, D. Smeets, D. Loeckx, G. Song, N. Tustison, B. Avants, J. C. Gee, M. Staring, S. Klein, B. C. Stoel, M. Urschler, M. Werlberger, J. Vandemeulebroucke, S. Rit, D. Sarrut, and J. P. Pluim. 
Evaluation of registration methods on thoracic CT: the EMPIRE10 challenge. IEEE Trans Med Imaging, 2011, 30(11):1901-20.

[23] K. W. Muir, J. Baird-Gunning, L. Walker, T. Baird, M. McCormick, and S. B. Coutts. Can the ischemic penumbra be identified on noncontrast CT of acute stroke?. Stroke, 2007, 38(9):2485-90.

[24] D. Vu, and M. H. Lev. Noncontrast CT in Acute Stroke. Seminars in ultrasound, CT, and MR, 2005, 26(6):380-386.

[25] D. Morhard, C. D. Wirth, M. F. Reiser, G. Schulte-Altedorneburg, and B. Ertl-Wagner. Optimal sequence timing of CT angiography and perfusion CT in patients with stroke. Eur J Radiol, 2013, 82(6):e286-9.

[26] M. Fisher, and G. W. Albers. Advanced imaging to extend the therapeutic time window of acute ischemic stroke. Ann Neurol, 2013, 73(1):4-9.

[27] K. N. Sheth, J. B. Terry, R. G. Nogueira, A. Horev, T. N. Nguyen, A. K. Fong, D. Gandhi, S. Prabhakaran, D. Wisco, B. A. Glenn, A. H. Tayal, B. Ludwig, M. S. Hussain, T. G. Jovin, P. F. Clemmons, C. Cronin, D. S. Liebeskind, M. Tian, and R. Gupta. Advanced modality imaging evaluation in acute ischemic stroke may lead to delayed endovascular reperfusion therapy without improvement in clinical outcomes. J Neurointerv Surg, 2013, 5 Suppl 1:i62-5. 


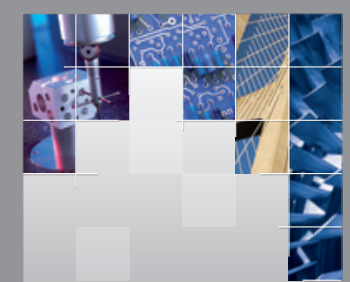

\section{Enfincering}
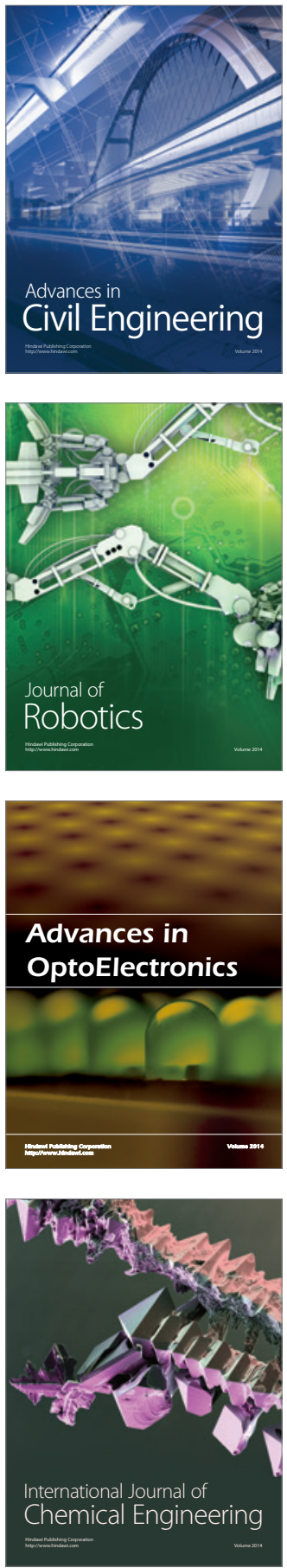

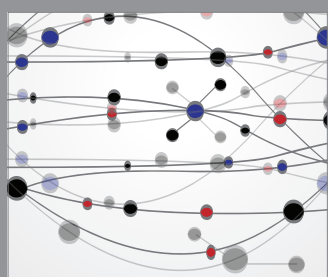

The Scientific World Journal

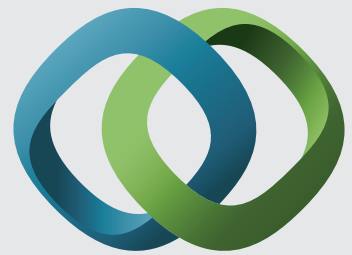

\section{Hindawi}

Submit your manuscripts at

http://www.hindawi.com
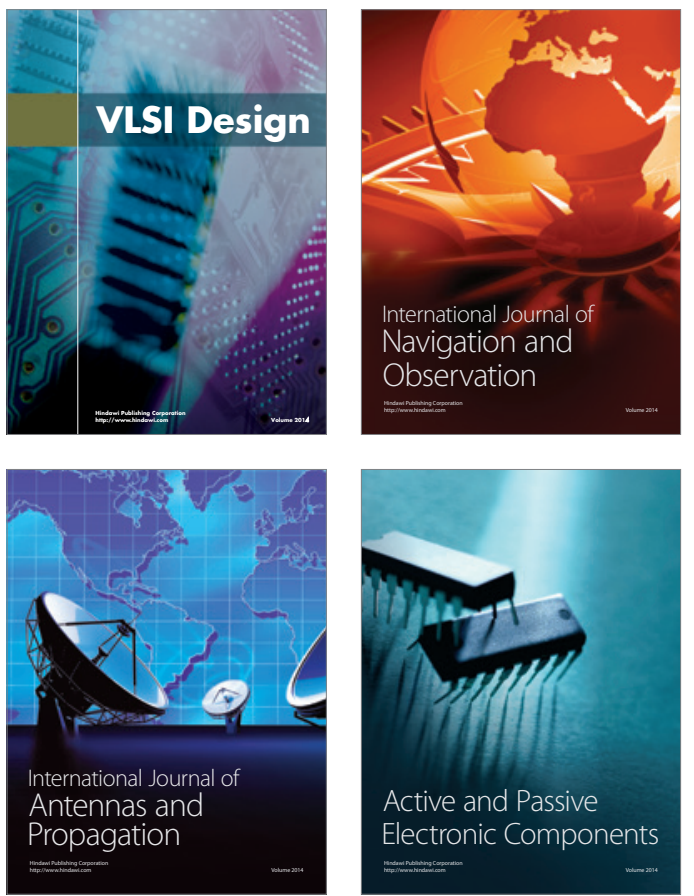
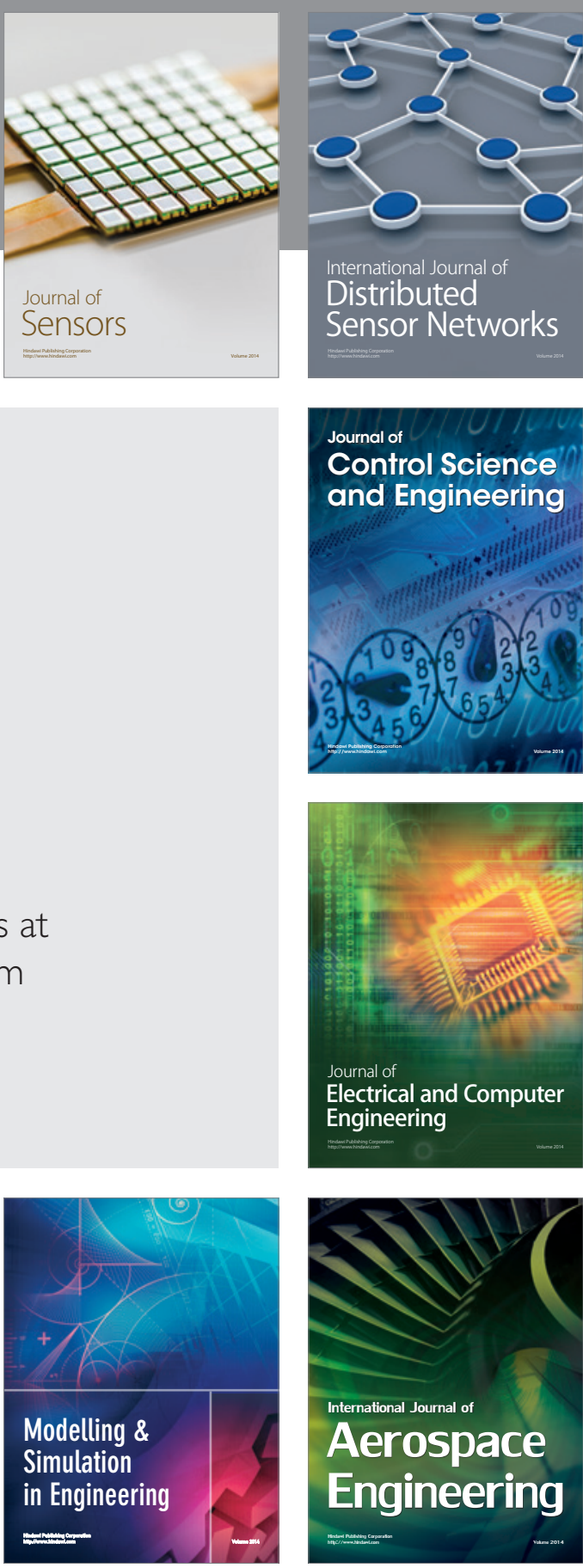

International Journal of

Distributed

Sensor Networks

Journal of

Control Science

and Engineering
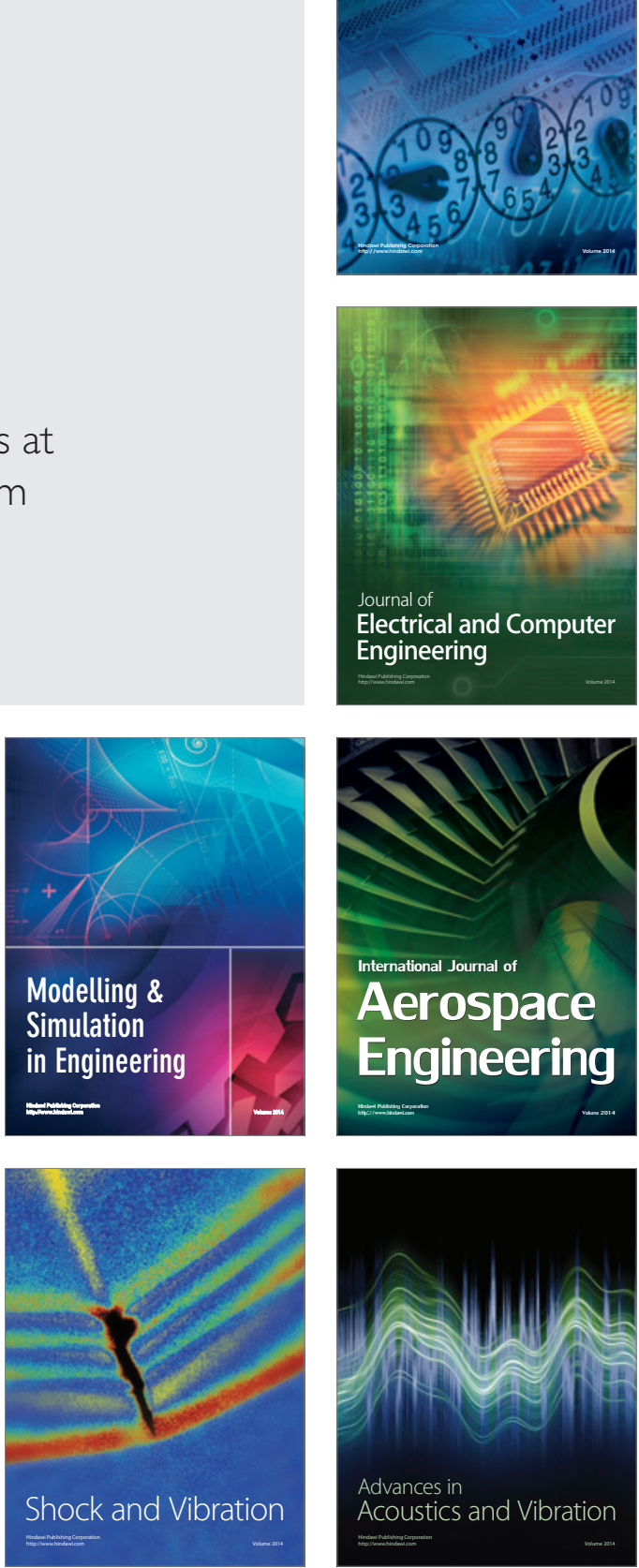